\title{
A UNITARY REPRESENTATION OF THE BASICAL CENTRAL EXTENSION OF A LOOP GROUP
}

\author{
RÉMI LÉANDRE
}

\begin{abstract}
We construct a measure over the string bundle associated to the loop space of a Riemannian manifold. We deduce a representation of a finite energy Kac-Moody group analoguous to the energy representation.
\end{abstract}

\section{Introduction}

Let $Q \rightarrow M$ be a principal bundle with structure group $G$. We suppose $M$ is compact and 2 connected. We suppose that $G$ is simple, compact and simply connected.

Let $L_{x}(M)$ and $L_{q}(Q)$ be the based loop space of $M$ and $Q: L_{q}(Q)$ is a principal bundle over $L_{x}(M)$ with structure group the based loop space of $G$. [Le ${ }_{5}$ ] has constructed over $L_{q}(Q)$ a measure by gluing together measures in the fiber and measure on the basis $L_{x}(M)$ : in the basis, [Le 5 ] has considered the Brownian bridge measure and in the fiber a measure over differentiable paths, which is convenient for the purpose of the spin representation of a loop group. Afterwards, $\left[\mathrm{Le}_{5}\right]$ defines a circle fiber bundle over $L_{q}(Q)$, when the first Pontryagin class of the bundle $Q \rightarrow M$ is equal to zero. In order to be more precise, the transition functionals over $L_{q}(Q)$ contain stochastic integrals: therefore $\left[\mathrm{Le}_{5}\right]$ has constructed this bundle by using the space of its functionals. It is possible to speak in [Le $]$ of the space of $L^{p}$ functionals. Moreover the basical central extension constituted in the basis of a loop group of loops which have two derivatives acts over the Hilbert space of $L^{2}$ functionals over this bundle.

The point of view presented here is different: instead of working over the based loop space of $Q$, we consider the free loop space of $Q$ called $L(Q)$. As a matter of fact, we consider continuous loops. Instead of constructing the measure over $L(Q)$ by starting from a measure over the basis and of measures over the fiber, we construct a measure globally. For that, we in-

Received March 8,1999.

2000 Mathematics Subject Classification: 58G32, 60H07. 
troduce a Riemannian structure over $Q$ such that the action of $G$ over $Q$ is an action by isometries. We consider over $L(Q)$ the B-H-K measure (See $\left.\left[\mathrm{Bi}_{2}\right],[\mathrm{H}-\mathrm{K}],\left[\mathrm{Le}_{1}\right],\left[\mathrm{Le}_{2}\right]\right) \mu$. We consider $L^{2}(\mu)$. The differentiable $C^{1}$ free loop group $L_{1}(G)$ acts over $L(Q)$ by: $q$. $\rightarrow$ q.g. This transformation keeps the measure quasi-invariant. We deduce a unitary representation of $L_{1}(G)$ over $L^{2}(\mu)$ which generalizes the Albeverio-Hoegh-Krohn representation of a loop group.

It is important for the purpose of the quantum field theory to construct the Dirac operator over the free loop space (See the survey of Segal [Se], the work of Witten $[\mathrm{Wi}]$ and the survey of Léandre $\left.\left[\mathrm{Le}_{10}\right]\right)$. For that, we need to construct a circle bundle over $L(Q), \tilde{L}(Q)$, such that we get a bundle over $L(M)$ whose structure group is the basical central extension $\tilde{L}_{1}(G)$ of $L_{1}(G)$. In this purpose, we construct a system of transition maps over $L(Q)$, which belong locally to all the Sobolev spaces (See $\left[\mathrm{Le}_{1}\right],\left[\mathrm{Le}_{2}\right]$ ) and we construct a circle bundle over $L(Q)$ by using the spaces of its functionals. We use for that a stochastic interpretation of the construction of Coquereaux-Pilch in the deterministic context (See $\left[\right.$ C.P], $\left[\mathrm{Le}_{4}\right]$ ). We define this lift $\tilde{L}(Q)$ by its functionals and introduce a formal measure $\tilde{\mu}$ over it and the associated Hilbert space $\tilde{L}^{2}(\tilde{\mu})$.

We have the following theorem:

TheOREM 1. $\tilde{L}_{1}(G)$ induces a unitary representation of $\tilde{L}^{2}(\tilde{\mu})$.

We thank M. Arnaudon and A. Vershik for helpfull discussions.

We thank for its warm hospitality the I.H.E.S. where this work was done.

\section{A unitary representation of the loop group}

Let $Q \rightarrow M$ be a principal bundle with compact structure group $G$ and compact basis $M$. Let us introduce over this principal bundle a connection $\nabla$. We split $T_{q}(Q)$ into the horizontal and the vertical vector spaces: this decomposition is kept under the right action of $G$. We will say that this gives an orthonormal decomposition of the tangent of $Q$ for a convenient Riemannian metric over $Q$. Since $G$ is compact, we suppose that this Riemannian metric is invariant under the action of $G$ over $Q$.

Let $\nabla^{Q}$ be the Levi-Civita connection over $Q$. Let $\Delta$ be the LaplaceBeltrami operator over $Q$. It has a heat semi-group represented by a heat kernel $p_{t}\left(q, q^{\prime}\right)$. Let $d P_{q, 1}$ the law of the Brownian bridge over $Q$ starting 
from $q$ and arriving in time 1 in $q$. We consider the measure over $L(Q)$, the free loop space of continuous applications from the circle $S^{1}$ into $Q$ :

$$
d \mu=\frac{p_{1}(q, q) d q \otimes d P_{1, q}}{\int p_{1}(q, q) d q}
$$

This measure is invariant under the natural circle action over $L(Q)$.

We denote by $q$. a typical element of $L(Q)$ and by $g$. a typical element of the free loop group of $C^{1}$ loops in $G$ denoted by $L_{1}(G)$.

Let $g$. be an element of $L_{1}(G)$. We deduce an action $\Psi_{g}: q$. $\rightarrow$ q.g. We have:

THEOREM 2. the law of q.g. under $\mu$ is equivalent to the law of $q$.

Proof. Let us suppose that $g$. $=g_{0}$. Since $g_{0}$ is an isometry, if $q$. follows the law of the Brownian bridge starting from $q_{0}$ and arriving in $q_{0}, q_{.} g_{0}$ follows the law of the Brownian bridge starting from $q_{0} g_{0}$ and arriving at $q_{0} g_{0}$. Moreover $p_{1}\left(q_{0} g_{0}, q_{0} g_{0}\right)=p_{1}\left(q_{0}, q_{0}\right)$ and the Jacobian of the map $q_{0} \rightarrow q_{0} g_{0}$ is equal to 1 . Therefore the total law is invariant. We can suppose $g_{0}=e$. It is the same to check that the law of the Brownian bridge starting from $q_{0}$ and arriving in $q_{0}$ is quasi-invariant under the transformation $q$. q.g.

It is the purpose of the quasi-invariance formula of Albeverio-HoeghKrohn ([A-H-K], [Sh], [Les] $)$. We identify an element $g$ of $G$ with the isometry $q \rightarrow q g$. Namely the parallel transport over $q . g$. is written as $\frac{\partial}{\partial q} g_{t} \tau_{t} O_{t}$ where $\tau_{t}$ is the parallel transport over $q$. for the Levi-Civita connection $\nabla^{Q}$ over $Q$, and $O_{t}$ is an adapted process of isometries in $T_{q_{0}}(Q)$ solution of the differential equation (See $\left.\left[\mathrm{Le}_{3}\right](2.5)\right)$ :

$$
d O_{t}=\tau_{t}^{-1}\left(\frac{\partial}{\partial q} g_{t}\right)^{-1}\left(-d / d t \frac{\partial}{\partial q} g_{t} \tau_{t} O_{t} d t-\Gamma_{\frac{\partial}{\partial q} g_{t} \tau_{t} O_{t}} d / d t g_{t} d t\right)
$$

where $O_{0}=I_{d}$ and where we have trivialized the tangent bundle by adding another bundle such that the Christoffel symbols $\Gamma$ are globally defined. We used too the Itô-Stratonovitch formula which says that:

$$
d g_{t}\left(q_{t}\right)=\frac{\partial}{\partial q} g_{t}\left(q_{t}\right) d q_{t}+\left(d / d t g_{t}\right)\left(q_{t}\right) d t
$$

Therefore

$$
\begin{aligned}
\tau_{t}^{-1}(\text { q.g. }) d(q . g .)_{t} & =O_{t}^{-1} \tau_{t}^{-1} d q_{t}+O_{t}^{-1} \tau_{t}^{-1} \frac{\partial}{\partial q} g_{t}^{-1}\left(d / d t g_{t}\left(q_{t}\right) d t\right. \\
& =C_{t} d B_{t}+H_{t} d t
\end{aligned}
$$


where $C_{t}$ is a semi-martingale of rotations in $T_{q_{0}}(Q)$ and $H_{t}$ a bounded previsible process. Let us remark that the isometry $q \rightarrow q g$ is denoted $q \rightarrow g(q)$ in order to be consistent with the traditional definition of function theory: the parenthesis means that we take the value of this isometry in $q$, and the absence of parenthesis means we take the action of the Lie group on the total space of the principal bundle. Since in the previous computation, we don't look at an action of a group over the total space of the principal bundle, but to a particular action of a fixed element of $G$, there is no confusion possible, if we add a parenthesis.

Let us do the same computations for the based path space of $Q$ : it is the space of continuous applications from $[0,1]$ into $Q$ endowed with the Brownian measure. Let us recall that $\tau_{t}^{-1} d q_{t}=d B_{t}$ is a Brownian motion in $T_{q_{0}}(Q)$. The law of $q$. and of $q . g$. over the path space are therefore equivalent. The quasi-invariance density denoted by $\exp \left[g\right.$.] belongs to all the $L^{p}$. We would like to disintegrate it over the Brownian bridge. Let us recall for that the differential Calculus over the based path space.

We consider as tangent vector field of a path $q$. the section of the tangent bundle over $q$. constituted by $\tau . H$. where $H$. is of finite energy and is a process in $T_{q_{0}}(Q)$ ([Bi 1$]$, [J.L]).

A cylindrical functional has a derivative $\langle d F, X\rangle=\int_{0}^{1} k(s) H_{s}^{\prime} d s$. We endow it with the Sobolev structure:

$$
\|F\|_{1, p}=E\left[\left(\int_{0}^{1} k(s)^{2} d s\right)^{p / 2}\right]^{1 / p}
$$

We can close the notion of a derivative of a functional in Sobolev spaces because we have the integration by parts $E[<d F, X>]=E[F \operatorname{div} X]$ if $X .=\tau . H$. and $H$. is deterministic $\left([\mathrm{Dr}],\left[\mathrm{Bi}_{1}\right]\right)$.

By using the connection

$$
\nabla X .=\tau \cdot D H
$$

where $D H$. is the $H$-derivative of $H$, we can define higher order Sobolev spaces: a functional $F$ belongs to these Sobolev spaces if

$$
\nabla^{k} F\left(X^{1}, . ., X^{k}\right)=\int . . \int k\left(s_{1}, . ., s_{k}\right) H_{s_{1}}^{\prime 1} . . H_{s_{k}}^{\prime k} d s_{1} . . d s_{k}
$$

and if the following Sobolev norms are finite:

$$
\|F\|_{k, p}=E\left[\left(\int . . \int k^{2}\left(s_{1}, . ., s_{k}\right) d s_{1} . . d s_{k}\right)^{p / 2}\right]^{1 / p}
$$


See $\left[\mathrm{Le}_{1}\right]$ and $\left[\mathrm{Le}_{2}\right]$ for more precisions. A functional which belongs to all the Sobolev spaces over the based path space can be restricted in a functional which belongs to all the Sobolev spaces over the based loop space, and its Sobolev norms over the based loop space can be estimated in terms of its Sobolev norms over the based loop space. exp $[g$. $]$ belongs to all the Sobolev spaces over the based path space. We deduce from this that $\sup _{q_{0}} E\left[\exp [g .]^{p}\right]^{1 / p}<\infty$ where we take the expectation over the based loop space starting from $q_{0}$. We deduce that the law of $q . g$. is absolutely continuous with the law of $q$. over the free loop space for $\mu$.

Let us introduce the map $\bar{\Psi}_{g}$. from $L^{2}(\mu)$ into himself:

$$
\bar{\Psi}_{g .}(F(q .))=\exp [g .]^{1 / 2}(q .) F(q . g .)
$$

for a functional $F$ belonging to $L^{2}(\mu)$.

THEOREM 3. $g . \rightarrow \bar{\Psi}_{g}$. is a unitary representation in $L^{2}(\mu)$ of $L_{1}(G)$.

\section{A unitary representation of a central extension of the loop group}

Let us suppose that $G$ is simple, simply connected and that $Q$ and $M$ are two connected. We construct a central extension $\tilde{L}_{1}(G)$ of $L_{1}(G)$ by considering the Kac-Moody form, which at the level of the Lie algebra of $L_{1}(G)$ satisfies to:

$$
c(X, Y .)=\frac{1}{2 \pi} \int_{0}^{1}<X_{s}, d Y_{s}>
$$

There is, since $G$ is simple simply connected, and since $H^{3}(G ; Z)=Z$ a smallest Killing product over the Lie algebra of $G$ such that $c / 2 \pi$ is a closed $Z$-valued two form over $L_{1}(G)$. We can construct $\tilde{L}_{1}(G)$ explicitly (See [C.P], [Mi], [P.S], [Ott]).

Let $e$. be the unit loop in $L_{1}(G)$. Let $l_{t}(g$. $)$ be a smooth path from $e$. to $g$. The bundle $\tilde{L}_{1}(G)$ is the set of $(l .(g),. \alpha) \alpha \in S^{1}$ submitted to the equivalence relation: $\left(l .\left(g_{.}\right), \alpha\right) \equiv\left(l^{\prime}\left(g^{\prime}\right), \alpha^{\prime}\right)$ if $g .=g^{\prime}$ and if

$$
\alpha^{\prime}=\exp \left[-i \int_{S} c\right] \alpha
$$

where $S$ is a surface with boundary the loop constituted of the path going from $e$. to $g$. by $l_{t}(g$. $)$ and coming back to $e$. by $l_{t}^{\prime}(g$. $)$ circled in the opposite sense. We can find such a surface because $L_{1}(G)$ is simply connected. 
Since $c / 2 \pi$ is closed $Z$-valued, the integral $\int_{S} c$ depends on $S$ by an integer multiplied by $2 \pi$. Therefore (3.2) has a meaning.

We define a group law over the bundle by putting:

$$
\left(l .\left(g_{.}\right), \alpha\right) .\left(l_{.}^{\prime}\left(g_{.}^{\prime}\right), \alpha^{\prime}\right)=\left(l .\left(g_{.}\right) * l_{.}^{\prime}\left(g_{.}^{\prime}\right), \alpha \alpha^{\prime}\right)
$$

where $l .(g). * l^{\prime}\left(g^{\prime}\right)$ is the path going from $e$. to $g . g^{\prime}$. in the following way: first we go from $e$. to $g$. by $l_{t}\left(g\right.$.) and afterwards we go from $g$. to $g . g^{\prime}$. by $g . l_{t}^{\prime}\left(g^{\prime}\right)$. It is the same to consider the couple of the path $l_{t}\left(g_{\text {. }}\right) l_{t}^{\prime}\left(g^{\prime}\right)$ and $\beta$ where

$$
\beta=\exp \left[-i \int_{S} c\right] \alpha \alpha^{\prime}
$$

$S$ is a surface bounded by the triangle $t \rightarrow l_{t}\left(g_{\text {. }}\right), t \rightarrow g l_{t}^{\prime}\left(g^{\prime}\right)$ and $t \rightarrow$ $l_{t}(g.) l_{t}^{\prime}\left(g^{\prime}\right)$, the last path being circled in the opposite direction.

(3.3) defines a group law. It is in particular compatible with the given equivalence relation. This allows us to define $\tilde{L}_{1}(G)$, the basical central extension of $L_{1}(G)$.

The problem is to find an Hilbert space $\tilde{L}^{2}(\tilde{\mu})$ where $\bar{\Psi}_{g}$. lifts in some sense in an action $\tilde{\Psi}_{\tilde{g}}$. of the central extension of the loop group.

Let $A_{Q}$ be a principal connection over $Q . A_{Q}$ is a Lie- $G$ valued 1 form on the total space. If $\tilde{\xi}_{q}$ is the fundamental vector field at $q \in Q$ corresponding to $\xi \in \operatorname{Lie} G$, we have:

$$
A_{Q}\left(\tilde{\xi}_{q}\right)=\xi
$$

The curvature $F_{Q}$ is given by:

$$
F_{Q}=d A_{Q}+1 / 2\left[A_{Q}, A_{Q}\right]
$$

Let us recall that:

$$
1 / 2\left[A_{Q}, A_{Q}\right](X, Y)=A_{Q}(X) A_{Q}(Y)-A_{Q}(Y) A_{Q}(X)
$$

We follow the construction of Coquereaux-Pilch ([C.P]). We introduce:

$$
\sigma_{Q}=\frac{1}{8 \pi^{2}}<A_{Q}, F_{Q}-1 / 6\left[A_{Q}, A_{Q}\right]>
$$

$d \sigma_{Q}=-\pi^{*} p_{1}(Q)$ where $p_{1}(Q)$ is the first Pontryagin class of $Q$ (represented by a form). Moreover, on the fiber:

$$
\sigma_{Q}(X, Y, Z)=\frac{1}{8 \pi^{2}}<X,[Y, Z]>=\sigma(X, Y, Z)
$$


We say that $\sigma_{Q}$ transgresses $\sigma$, the canonical 3 form over the fiber.

We consider the transgression over the free loop space of the total space of $\sigma_{Q}$. It is given by:

$$
\tau\left(\sigma_{Q}\right)\left(X_{.}, Y_{.}\right)=\int_{0}^{1} \sigma_{Q}\left(d q_{s}, X_{s}, Y_{s}\right)
$$

(We work over the finite energy loop space over $Q$, such that a tangent vector over a loop $q$. is a section of finite energy in the tangent bundle of $Q$ over $q . \cdot$.).

The last ingredient of $[$ C.P $]$ is to produce a 2 -form $\omega_{Q}$ over $L_{1}(Q)$, the space of finite energy loops in $Q$, which is equal to $c$ in the fiber, modulo a normalizing term. It is:

$$
\omega_{Q}^{\prime}=\frac{1}{2 \pi} \int_{0}^{1}\left(1 / 2<A_{Q}, d / d t A_{Q}>-<F_{Q}, A_{Q}\left(d q_{s}\right)>\right)
$$

$d / d t A_{Q}$ is the form which to a vector $X_{t}$ over $q$. associates the time derivative in $t$ of $A_{Q}\left(q_{t}\right)\left(X_{t}\right)$.

We have $([\mathrm{C} . \mathrm{P}])$ :

$$
\frac{1}{2 \pi} \omega_{Q}^{\prime}=\tau\left(\sigma_{Q}\right)-d\left(\frac{1}{8 \pi^{2}} \int_{0}^{1}<A_{Q}, A_{Q}\left(d q_{s}\right)>\right)
$$

We do the following hypothesis:

Hypothesis. $p_{1}(Q)=0$ in cohomology.

By the theory of Chern-Simons form, if $p_{1}(Q)=0$, in cohomology, it is possible to find a representative $\nu$ such that $-p_{1}(Q)=d \nu$ and such that $\sigma_{Q}-\pi^{*} \nu$ is a $Z$-valued form over $Q([\mathrm{C} . \mathrm{S}]$ Theorem 3.10).

Let us introduce the form:

$$
\frac{\omega_{Q}}{2 \pi}=\frac{\omega_{Q}^{\prime}}{2 \pi}-\tau\left(\pi^{*} \nu\right)
$$

$\omega_{Q} / 2 \pi$ is closed $Z$-valued over $L_{1}(Q)$. Since $L_{1}(Q)$ is simply connected, it defines a unique circle bundle whose curvature is $i \omega_{Q}$. Our purpose is to generalize this result in the stochastic context.

Let $q_{\text {ref,. }}$ be a finite energy loop of reference in $Q$. Let $q_{i, \text {. }}$ be finite energy loops in $Q$ such that the union of balls $B\left(q_{i,} ; \delta\right)$ for the uniform distance is equal to $L(Q)$. 
If $q$. belongs to $B\left(q_{i,} ; \delta\right)$, there is a distinguished curve joining $q$. to $q_{i, .}$; it is

$$
l_{i, t}\left(q_{.}\right)_{s}=\exp _{q_{i, s}}\left[t\left(q_{s}-q_{i, s}\right)\right]
$$

(Let us recall that we have over $Q$ a Riemannian metric such that the exponential map are defined and are local bijection. Moreover, $\left(q_{s}-q_{i, s}\right)$ denotes the vector of the unique geodesic joining $q_{i, s}$ to $q_{s}$.) We join by $l_{i, t}(q$. $) q$. to $q_{i, .}$. We choose a path joining $q_{i, .}$ to $q_{\text {ref,.. }}$. We glue the two paths. We get a path joining $q$. to $q_{\text {ref,. }}$ still denoted by $l_{i, .}(q$. $)$.

Let $q$. belonging to $B\left(q_{j,} ; \delta\right)$ too. We produce another distinguished curve $l_{j, .}(q$.$) . We can find a surface whose boundary is the curve l_{i, .}(q$. $)$ circled in the direct sense and the curve $l_{j,}(q$. $)$ circled in the opposite sense. We fulfill the small stochastic triangle constituted by $l_{i, .}(q),. t \in[0,1], l_{j, .}(q$.), $t \in[0,1]$ and by the segment constituted by the exponential charts joining $q_{i, \text {. to }} q_{j, .}$. It is possible to use a system of exponential in order to produce such a small stochastic surface, because we have supposed $\delta$ enough small (See $\left[\mathrm{Le}_{4}\right](1.7)$ ). And we find, since $L_{1}(Q)$ is supposed simply connected, a big deterministic surface whose boundary is the triangle $q_{i, .}, q_{j, .}, q_{\text {ref,.. }}$ We deduce a distinguished surface whose boundary is constituted of path $l_{i, .}(q$.) circled in the direct sense and of $l_{j, .}(q$.$) circled in the opposite sense. We$ denote one generic element of this distinguished surface by $l_{u, v, i, j}(q$.).

We put:

$$
\rho_{i, j}(q .)=\exp \left[-i \int_{S_{i, j}(q .)} \omega_{Q}\right]
$$

The integral of $\tau\left(\pi^{*} \nu\right)$ over $S_{i, j}(q$. $)$ does not put any difficulty and leads as in $\left[\mathrm{Le}_{6}\right]$ to some non anticipative Stratonovich integrals. The only term which put some difficulty is the term $d / d t A_{Q}$. First of all, we take the differential in $s$ of $s \rightarrow l_{u, v, i, j}(q .)_{s}$, which is a semi-martingale and which leads to a Stratonovich integral. The term where we use the time differential of $(\partial / \partial u)\left(l_{u, v, i, j}(q .)_{s}\right)$ leads too to a non anticipative Stratonovich integral, because $s \rightarrow(\partial / \partial u)\left(l_{u, v, i, j}(q .)_{s}\right)$ is a semi-martingale over the semi-martingale $s \rightarrow l_{u, v, i, j}(q .)_{s}$.

Moreover, $q$. $\rightarrow \rho_{i, j}(q$. $)$ belongs to all the Sobolev spaces. It can be seen as follows: we plung $Q$ in $R^{d}$, we extend $A, F$ and $\pi^{*} \nu$ to $R^{d}$, and we extend to $R^{d} \times R^{d}$ the map $(x, y) \rightarrow \exp _{x}(y)$. Therefore we extend to all $L(Q)$ the surface $S_{i, j}\left(q\right.$.), and we extend the integral $\int_{S_{i, j}(q .)} \omega_{Q}$ to all 
$L(Q)$. This shows us that $\rho_{i, j}(q$. $)$ is the restriction to $B\left(q_{i, .} ; \delta\right) \cap B\left(q_{j, .} ; \delta\right)$ of an application with values in $S^{1}$ which belongs to all the Sobolev spaces. Moreover, by the properties of the Stratonovich integral, if $q^{n}$ is the polygonal approximation of $q$., which is defined if the length of the subdivision is big enough, we get almost surely:

$$
\rho_{i, j}\left(q^{n}\right) \rightarrow \rho_{i, j}(q .)
$$

Moreover, $\rho_{i, j}\left(q_{.}^{n}\right) \rho_{j, k}\left(q^{n}\right) \rho_{k, i}\left(q^{n}\right)=1$ and $\rho_{i, j}\left(q^{n}\right) \rho_{j, i}\left(q_{.}^{n}\right)=1$. This shows that almost surely over $B\left(q_{i,} ; \delta\right) \cap B\left(q_{j} ; \delta\right) \cap B\left(q_{k, .} ; \delta\right)$ :

$$
\rho_{i, j}(q .) \rho_{j, k}(q .) \rho_{k, i}(q .)=1
$$

and

$$
\rho_{i, j}(q .) \rho_{j, i}(q .)=1
$$

So we cannot define the topological space of the circle bundle associated to $\omega_{Q}$. But we can define its space of $L^{2}$ functionals.

A functional of $\tilde{L}(Q)$ is given by the following data:

A system of applications from $B\left(q_{i,} ; \delta\right) \times S^{1} \rightarrow R$ called $\tilde{F}_{i}\left(q ., u_{i}\right)$ satisfying to the relation $\tilde{F}_{j}\left(q ., u_{j}\right)=\tilde{F}_{i}\left(q ., u_{i}\right)$ if $u_{j}=\rho_{j, i}(q.) u_{i}$ almost surely.

Over the fiber, we put:

$$
\|\tilde{F}\|_{p, q .}=\left(\int_{S^{1}}\left|F_{i}\left(q ., u_{i}\right)\right|^{p} d u_{i}\right)^{1 / p}
$$

Since the Haar measure over the circle is invariant under rotation, (3.20) is compatible with the change of trivialization.

We put

$$
\|\tilde{F}\|_{\tilde{L}^{2}}=E\left[\|\tilde{F}\|_{2, q .}^{2}\right]^{1 / 2}=\tilde{E}\left[|\tilde{F}|^{2}\right]^{1 / 2}
$$

We define as that an Hilbert space $\tilde{L}^{2}(\tilde{\mu})$.

Let $q . \in B\left(q_{i, .} ; \delta\right)$ and $l_{i, .}(q$. $)$ be the associated distinguished path. Let

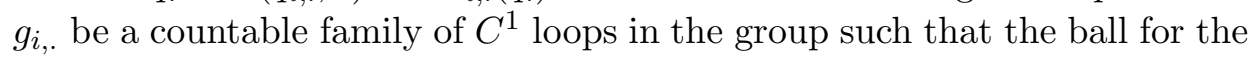
uniform distance $B\left(g_{i,} ; \delta\right)$ constitute a cover of $L_{1}(G)$. Let $g . \in B\left(g_{i,} ; \delta\right)$. We can construct as before a curve which joins $g$. to $g_{i,}$, by using the exponential of a Lie group instead of the exponential of the Riemannian

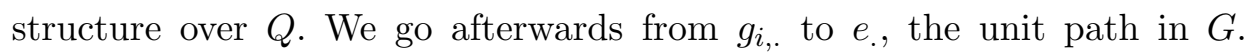
We produce by this procedure a distinguished curve joining $g$. to $e$, called 
$l_{i, .}(g$.$) . An element of \tilde{L}(Q)$ is the couple of the distinguished curve joining $q_{\text {ref,. }}$ to $q_{\text {. }} l_{i, .}\left(q_{\text {. }}\right)$ and of $\alpha$ when $q . \in B\left(q_{i, .} ; \delta\right)$. Let us denote $\left(l_{i, .}(q),. \alpha\right)=\tilde{q}_{\text {. }}$. $\tilde{g}_{.}=\left(l_{j, .}\left(g_{.}\right), \beta\right)$ acts as follows over $\tilde{q}_{\text {. }}$.

We consider the curve $l_{i, .}\left(q_{.}\right) l_{j, .}\left(g_{.}\right)$, and the couple of $\left(l_{i, .}\left(q_{.}\right) l_{j, .}\left(g_{\text {. }}\right)\right.$, $\left.\exp \left[-i \int_{S_{1}\left(q_{.}, g_{.}\right)} \omega_{Q}\right] \alpha \beta\right)$ where $S_{1}\left(q_{.}, g_{\text {. }}\right)$ is the surface $l_{i, t}\left(q_{.}\right) l_{j, s}\left(g_{.}\right) ; s \leq t$ and where $\omega_{Q}$ is integrated over this distinguished surface by using the theory of Stratonovitch integral. Let us suppose that $q . g$. belongs to $B\left(q_{k, .} ; \delta\right)$. We can find a distinguished surface $S\left(q ., g_{\text {. }}\right)$ with boundary $l_{i, .}(q.) l_{j, .}\left(g_{\text {. }}\right)$ and $l_{k, .}\left(g_{. q}\right.$.) such that we can integrate $\omega_{Q}$ over $S(q ., g$.$) by using the the-$ ory of stochastic integrals (Let us recall that $q$. is random and that $g$. is deterministic). We proceed as before in order to do that: we find a small stochastic surface whose boundary is the small stochastic triangle constituted of $q_{.} ., q_{i, .} g_{j,}$ and $q_{k,}$. by using a system of exponential maps. And we find a big deterministic surface whose boundary is the big deterministic triangle constituted of $q_{i, .} g_{j, .}, q_{k, .}$ and $q_{\text {ref,.. }}$.

We identify $\left(l_{i, .}(q.) l_{j, .}(g),. \exp \left[-i \int_{S_{1}\left(q_{.}, g .\right)} \omega_{Q}\right] \alpha \beta\right)$ with $\left(l_{k, .}\left(q . g_{.}\right)\right.$, $\left.\exp \left[-i \int_{S(q ., g .)} \omega_{Q}\right] \alpha \beta\right)=\tilde{q} . \tilde{g}_{\text {. }}$

Let $\pi$ the application which to $\tilde{q}$. associates the end of the path which represents it. Let $\pi$ the application which to $\tilde{g}$. associates the end of a path which represents $\tilde{g}_{\text {. }}$.

We put:

$$
\tilde{\Psi}_{\tilde{g} .}(\tilde{F})(\tilde{q} .)=\left\{\frac{d \mu(\pi \tilde{q} . \pi \tilde{g} .)}{d \mu(\pi \tilde{q} .)}\right\}^{1 / 2} \tilde{F}(\tilde{q} . \tilde{g} .)
$$

where this expression is defined in local charts by previous rules. We check (See $[$ C.P]) that these local transformations are compatible with all the equivalence relation, and that it gives a group action of $\tilde{L}_{1}(G)$. Moreover the formal law of $\tilde{q} . \tilde{g}$. is equivalent to the formal law of $\tilde{q}$.: namely the law of $\pi \tilde{q}$. $\pi \tilde{g}$. is equivalent to the law of $\tilde{g}$. (See chapter II). And the action of $\tilde{g}$. induces a multiplication in the fiber, which keeps the Haar measure invariant.

We deduce:

THEOREM 4. $\tilde{g}_{\text {. }} \rightarrow \tilde{\Psi}_{\tilde{g}}$. induces a unitary representation of $\tilde{L}_{1}(G)$ over $\tilde{L}^{2}(\tilde{\mu})$.

Remark. It can be seen that $\omega_{Q}$ is a stochastic form for the stochastic diffeology of [Le9] over the free loop space of the total space of the principal 
bundle. So the circle bundle considered here, whose fiber is almost surely defined, is isomorphic to a true bundle over the Hölderian loop space. The problem is that this true bundle is given by an abstract argument, and that we cannot do explicit computation over it.

\section{REFERENCES}

[A.H-K] S. Albeverio and R. Hoegh-Krohn, The energy representation of Sobolev Lie groups, Compositio Math., 36 (1978), 37-52.

[A.H-K.T.V] S. Albeverio, R. Hoegh-Krohn, D. Testard, A. Vershik, Factorial representation of path groups, J. Funct. Analysis, 51 (1983), 115-131.

$\left[\mathrm{Bi}_{1}\right] \quad$ J. M. Bismut, Large deviations and the Malliavin Calculus, Progress in Maths., 45, Birkhäuser, 1984.

$\left[\mathrm{Bi}_{2}\right]$ J. M. Bismut, Index theorem and equivariant cohomology on the loop space, Com. Maths. Physics., 98 (1985), 213-237.

[C.M] A. L. Carey and M. K. Murray, String structure and the path fibration of a group, Com. Math. Physics, 141 (1991), 441-452.

[C.S] S. S. Chern and J. Simons, Characteristic forms and geometric invariants, Ann. maths., 99 (1974), 48-69.

[C.P] R. Coquereaux and K. Pilch, String structures on loop bundles, Com. Maths. Physics., 120 (1989), 353-378.

[Dr] B. Driver, A Cameron-Martin type quasi-invariance formula for Brownian motion on compact manifolds, J. Funct. Analysis, 110 (1992), 272-376.

[H-K] R. Hoegh-Krohn, Relativistic quantum statistical mechanics in 2 dimensional space time, Com. Math. Physics, 38 (1974), 195-224.

[J.L] J. D. S. Jones and R. Léandre, $L^{p}$ Chen forms on loop spaces, In "Stochastic Analysis" (1991), Cambridge University Press, 104-162.

[Ka] V. Kac, Infinite dimensional Lie algebras, Cambridge University Press, 1985.

[K] T. Killingback, World-sheet anomalies and loop geometry, Nucl. Phys. B., 288 (1987), 578-588.

[Le $\mathrm{Le}_{1} \quad \mathrm{R}$. Léandre, Integration by parts and rotationnaly invariant Sobolev Calculus on free loop spaces, XXVII Winter School of theoretical physics, J. Geometry and Physics, 11 (1993), 517-528.

[Le $\left.\mathrm{Le}_{2}\right]$ Invariant Sobolev Calculus on free loop space, Acta Applicandae Mathematicae, 46 (1997), 267-350.

[Le 3 _ Brownian cohomology of an homogeneous manifold, In "New trends in stochastic analysis" (1997), World Scientific, 305-347.

[Le $\mathrm{Le}_{4} \quad$ Hilbert space of spinor fields over the free loop space, Reviews in Maths. Physics, 9.2 (1997), 243-277.

[Le $\mathrm{Le}_{5} \quad$ Stochastic gauge transform of the string bundle, J. Geometry and Physics, 26 (1998), 1-25. 
[Le $] \quad$ String structure over the Brownian bridge, J. Math. Physics, 40.1 (1999), 454-479.

[Le 7 Stochastic cohomology of the frame bundle of the loop space, J. Nonlinear Math. Physics, 5.1 (1998), 23-40.

[Les] Singular integral homology of the stochastic loop space, Infi. Dim. Analysis, Quantum Proba. rel. Topics, 1.1 (1998), 17-31.

[Le9] Stochastic cohomology of Chen-Souriau and line bundle over the Brownian bridge, to be published in P.T.R.F.

[Le $\left.{ }_{10}\right]$ C Cover of the Brownian bridge and stochastic symplectic action, Rev. Math. Phys., 12 (2000), no. 1, 91-137.

[M] P. Malliavin, Stochastic Analysis, Grund. Math. Wissen, 313, Springer, 1997.

[M.M] M. P. Malliavin and P. Malliavin, Integration on loop groups III. Asymptotics Peter-Weyl orthogonality, J. Funct. Analysis, 108 (1992), 13-46.

[Mi] J. Mickelsson, Current algebras and groups, Plenum Press, 1989.

[Ott] J. T. Ottesen, Infinite dimensional groups and algebras in Quantum physics, Lect. Notes. Physics, 1995.

[P.S] A. Pressley and G. Segal, Loop groups, Oxford University Press, 1986.

[Se] G. Segal, Elliptic cohomology, Séminaire Bourbaki 695, Astérisque 161-162 (1988), 187-201.

[Wi] E. Witten, The index of the Dirac operator in loop space, In "elliptic curves and modular forms in algebraic topology", Lec. Notes. Maths., 1326 (1988), 161-181.

Département de Mathématiques

Institut Elie Cartan

Faculté des Sciences

Université de Nancy I

54000. Vandoeuvre-les-Nancy

France

Département de Mathématiques

I.H.E.S

91440. Bures sur Yvette

France 